\title{
El derecho de la mujer a la vivienda en España (2008-2017)
}

\author{
Jordi Bosch Meda' (iD 0000-0001-7678-0953 \\ 'Universitat Politècnica de Catalunya, Escola Tècnica Superior d'Arquitectura de \\ Barcelona, Barcelona, España. 08028 -informacio.etsab@upc.edu
}

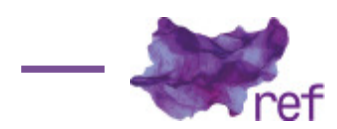

Resumen: El principal objetivo de este artículo es analizar la dimensión económica de la exclusión residencial entre las mujeres en España durante el periodo 2008-2017. A pesar de que el derecho de la mujer a una vivienda digna y la necesidad de desarrollar políticas para su cumplimiento gozan de un amplio reconocimiento en la agenda internacional, existe un abismo entre este y la realidad. En España, el estallido de la burbuja inmobiliaria en 2008 y la posterior recesión económica provocaron una grave crisis residencial que ha afectado con mayor intensidad a las mujeres, sobre todo, a las que viven solas y las jefas de familia de hogares monomarentales. Esta feminización de la exclusión residencial en España puede constatarse en la relación entre ingresos del hogar y costes de la vivienda, así como en la incidencia de los desahucios y las ejecuciones hipotecarias entre hombres y mujeres.

Palabras clave: derecho a la vivienda; política de vivienda; perspectiva de género; exclusión residencial; desahucios.

\section{O direito das mulheres à habitação em Espanha (2008-2017)}

Resumo: O principal objetivo deste artigo é analisar a dimensão econômica da exclusão habitacional entre as mulheres na Espanha durante o período 2008-2017. O direito das mulheres à moradia adequada e a necessidade de desenvolver políticas para seu cumprimento gozam de amplo reconhecimento na agenda internacional. No entanto, há uma diferença entre este reconhecimento e a realidade. Na Espanha, o estouro da bolha imobiliária em 2008 e a subsequente recessão económica causaram uma grave crise habitacional que afetou as mulheres mais profundamente, especialmente às famílias monomarentais e as mulheres que vivem sozinhas. Esta feminização da exclusão habitacional na Espanha pode constatar-se ao comparar-se a relação entre os custos de habitação e a renda familiar, e na incidência de despejos e execuções hipotecárias entre homens e mulheres.

Palavras-chave: direito à habitação; política de habitação; perspectiva de gênero; exclusão habitacional; despejos.

\section{Women's right to housing in Spain (2008-2017)}

Abstract: The main aim of this article is to examine the economic dimension of residential exclusion among women in Spain during the 2008-2017 period. The women's right to adequate housing and the need to develop policies in this regard are widely recognized on the international agenda. However, there is an abyss between recognition and reality. In Spain, the burst of the real estate bubble in 2008 and the subsequent economic recession caused a severe housing crisis that affected women more than men, particularly single-mother households and female single-person households. The feminization of housing exclusion in Spain can be seen in the share of total housing costs in the total disposable household income, as well as in the incidence of evictions and foreclosures between men and women. Keywords: Right to Housing; Housing Policy; Gender Perspective; Housing Exclusion; Evictions.

\section{Introducción}

En la segunda mitad de los noventa, el sector residencial español inició un periodo de fuerte expansión que estuvo caracterizado por un intenso aumento del precio de la vivienda, y por un alto ritmo de construcción de nuevas unidades residenciales y de operaciones de compraventa. 
Sin embargo, a finales de 2007 el ciclo económico se invirtió, la economía española entró en recisión y el desempleo empezó a subir con fuerza (Tabla 1). Lógicamente, el mercado de vivienda frenó bruscamente, los impagos se multiplicaron y, con ellos, los desahucios y las ejecuciones hipotecarias, sobre todo en las periferias metropolitanas y áreas turísticas que experimentaron una mayor intensidad constructora durante la fase alcista (ODESC/PAH, 2013; Ricardo MÉNDEZ; Luis ABAD; Julio PLAZA, 2014; AMNISTíA INTERNACIONAL ESPAÑA, 2015, 2017; Ricardo MÉNDEZ; Julio PLAZA, 2016; Ricardo MÉNDEZ, 2017; Eduard SALA, 2018).

Hoy, cerca de diez años después del estallido de la burbuja inmobiliaria, el problema ha evolucionado, pero dista mucho de estar resuelto. En los juzgados españoles aún hay miles de expedientes de ejecuciones hipotecarias pendientes de resolución (MÉNDEZ; ABAD; PLAZA, 2014), y la morosidad en el mercado de alquiler sigue en valores muy elevados. Según datos del Consejo General del Poder Judicial, entre 2013 y 2015 se produjeron cada año más de 67 mil lanzamientos; en 2016, unos 63 mil, de los cuales, un 54\% tuvieron lugar por el impago del alquiler; y en 2017 , de los más de 60 mil, cerca de un $59 \%$ fueron por el mismo motivo.

Como cabía esperar, los hogares socioeconómicamente más vulnerables, muchos de los cuales están encabezados por una mujer a causa de las múltiples formas de discriminación que padecen, han sido los que en mayor proporción y de un modo más grave están padeciendo esta crisis residencial.

En este contexto, el objetivo de este artículo es analizar la dimensión económica de la exclusión residencial entre las mujeres en España durante el periodo 2008-2017, así como examinar los principales factores que determinan dicha exclusión, a saber, su integración laboral, y el alcance de las políticas de vivienda y de igualdad llevadas a cabo.

La principal aportación de esta investigación radica en el hecho de que ofrece un retrato completo (en lo relativo a datos estadísticos empleados, estudios revisados y aspectos considerados) y temporalmente actualizado de la feminización de la exclusión residencial que está sufriendo un creciente segmento de la sociedad española tras el estallido de la burbuja.

\section{El derecho de la mujer a la vivienda en el plano internacional}

La necesidad de incorporar la perspectiva de género en las políticas de vivienda para corregir su peor situación en los mercados residenciales goza desde hace tiempo de un amplio reconocimiento en la agenda política internacional. Uno de los primeros documentos que lo incluye es el Pacto Internacional de Derechos Económicos, Sociales y Culturales (PIDESC) de 1966. Éste, ratificado por España en 1976, insta a los estados a asegurar que los hombres y las mujeres gocen de igual modo de todos los derechos económicos, sociales y culturales enunciados en ese Pacto, entre los que se incluye el derecho de toda persona a una vivienda adecuada.

La Convención sobre la Eliminación de Todas las Formas de Discriminación contra la Mujer (CEDAW) adoptada por Naciones Unidas en 1979, en vigor como tratado internacional desde septiembre de 1981 y también ratificado por España en 1984, así como las resoluciones del Comité CEDAW que supervisa su cumplimiento constituyen otro referente. Entre otros aspectos, este tratado exhorta a los estados a adoptar todas las medidas apropiadas, inclusive la provisión de una vivienda adecuada cuando sea necesario, para eliminar cualquier situación discriminatoria que pueda sufrir la mujer.

La Cuarta Conferencia Mundial sobre la Mujer, de 1995, en la que se aprobó la Plataforma de Acción de Beijing, supuso otro importante avance por el amplio desarrollo que recibe el problema residencial de la mujer. Entre las resoluciones acordadas, consta el deber de los gobiernos de facilitar a las mujeres viviendas a precios razonables y el de eliminar todos los obstáculos que impiden su acceso a la vivienda y a la tierra.

Todas las conferencias internacionales de Naciones Unidas sobre los asentamientos humanos, más conocidas como Hábitat, también han planteado el problema de la exclusión residencial de las mujeres. En la primera, celebrada en Vancouver en 1976 (Hábitat I), se instó a todos los países a crear las condiciones para la plena integración de las mujeres en el plano político, económico y social, sobre todo en los proyectos relativos a asentamientos humanos. En la segunda, que tuvo lugar en Estambul en 1996 (Hábitat II), se hizo hincapié en la necesidad de: (i) alcanzar la plena igualdad entre hombres y mujeres; (ii) prestar especial atención a los problemas de exclusión y pobreza que sufre la mujer; (iii) conseguir que la mujer participe plenamente, en condiciones de igualdad en todas las esferas sociales y políticas, en particular, en las políticas urbanas desde los gobiernos locales; (iv) garantizar la protección jurídica de la igualdad entre hombres y mujeres en materia de derechos a la tierra y a la propiedad; ( $v$ ) y de promover medidas contra la delincuencia y la violencia urbanas que, con mayor frecuencia, padecen las mujeres. Veinte años más tarde, en Quito, en la tercera conferencia (Hábitat III), se adoptó la denominada Nueva Agenda Urbana (NAU). La NAU define los parámetros que deben regir el desarrollo de las ciudades en los próximos 20 años. Respecto la mujer, la NAU conmina a los países firmantes a "promover el desarrollo de políticas y enfoques habitacionales integrados que tengan en cuenta la edad y el género" (NACIONES UNIDAS, 2017, p. 22). 
También la Agenda 2030 de Desarrollo Sostenible, aprobada en 2015 por 197 países, entre los que se incluye España, y en vigor desde principios de 2016, contempla, entre sus objetivos, "Lograr la igualdad entre los géneros y empoderar a todas las mujeres y las niñas" (objetivo de desarrollo sostenible número 5), así como "Lograr que las ciudades y los asentamientos humanos sean inclusivos, seguros, resilientes y sostenibles" (objetivo 11), para lo cual, plantea, entre otras metas, "Aprobar y fortalecer políticas acertadas y leyes aplicables para promover la igualdad de género y el empoderamiento de todas las mujeres y las niñas a todos los niveles" y "asegurar el acceso de todas las personas a viviendas y servicios básicos adecuados, seguros y asequibles y mejorar los barrios marginales".

En el contexto europeo, son numerosas las referencias de la legislación comunitaria al principio de igualdad entre hombres y mujeres. El Tratado fundacional de Roma de 1957 lo consagró en materia laboral, el Tratado de Ámsterdam de 1997atribuyó a la Unión Europea la misión de promover la igualdad de oportunidades entre hombres y mujeres, y, diez años más tarde, el de Lisboa precisó que uno de los valores comunes a todos los estados miembros era la igualdad entre mujeres y hombres.

Sin embargo, las iniciativas internacionales que con mayor detalle han abordado el problema de las necesidades residenciales de la mujer han surgido en torno al papel de los gobiernos locales en materia de derechos humanos y políticas urbanas. La Carta Europea de Salvaguarda de los Derechos Humanos en la Ciudad (Saint-Denis, 2000) es una buena muestra de ello. Suscrita por 175 ciudades españolas, entre las que se encuentran Madrid, Barcelona, Valencia, Donostia- San Sebastián, Sevilla, y Vitoria-Gasteiz, en su artículo XVI sobre el Derecho a la vivienda precisa que:

Las autoridades municipales velan por la existencia de una oferta adecuada de vivienda y equipamientos de barrio para todos sus ciudadanos y ciudadanas, sin distinción por razón del nivel de ingresos. Dichos equipamientos deben comprender estructuras de acogida para los sin techo que garanticen su seguridad y su dignidad, y estructuras para las mujeres víctimas de la violencia, en particular de la violencia doméstica, malos tratos, y para las que intentan salir de la prostitución. (CGLU, 2012, p. 51)

Tras esta carta, se han sucedido numerosas iniciativas similares como, por ejemplo, la Carta Mundial del Derecho a la Ciudad propuesta por los movimientos sociales reunidos en Porto Alegre en el primer Foro Social Mundial en el 2001, la Carta de Derechos y Responsabilidades de Montreal del año 2007, la Carta de la Ciudad de México por el Derecho a la Ciudad del 2010, o la CartaAgenda Mundial de Derechos Humanos en la Ciudad adoptada en Florencia a finales de 2011 bajo el impulso de la plataforma mundial Ciudades y Gobiernos Locales Unidos (CGLU). Todas ellas, de un modo u otro, abogan por la inclusión de la perspectiva de género en las políticas urbanas, en especial, en las de vivienda.

No obstante, a pesar de este vasto reconocimiento del derecho de la mujer a la vivienda y del deber de avanzar en su efectividad, lamentablemente, la exclusión residencial de la mujer es una realidad en muchos países. Los sucesivos informes del Relator Especial de Naciones Unidas sobre el derecho de la mujer a una vivienda adecuada (Miloon KOTHARI, 2003, 2004, 2006), los de las relatoras posteriores sobre la vivienda adecuada como elemento integrante del derecho a un nivel de vida adecuado (Raquel ROLNIK, 2011; Leilani FARHA, 2015), así como los publicados directamente por Naciones Unidas (UN-HUMAN RIGHTS, 2012; UN-HABITAT, 2014) y los relativos a las consultas regionales sobre este asunto (UN-HABITAT, 2002, 2004, 2005a, 2005b, 2006; ODESC, 2006; María Silvia EMANUELLI, 2004) denuncian que las mujeres sufren un trato discriminatorio en todas las esferas esenciales para el disfrute de una vivienda adecuada a causa de un cúmulo de factores interrelacionados, entre los que cabe destacar: (i) el desigual acceso de la mujer a la educación y a las fuentes de ingresos;(ii) su inferior posición en los mercados de trabajo; (iii) sus mayores tasas de pobreza y exclusión social,(iv) las frecuentes barreras legales y sociales para acceder a la tierra, a la propiedad, a la herencia, al crédito y a los subsidios de vivienda; ( $v$ ) y la ausencia de programas nacionales para corregir esta discriminación. Además, estos informes identifican unos grupos de mujeres en una especial situación de vulnerabilidad en materia de vivienda debido a la intersección de diferentes ejes de exclusión: los hogares monomarentales', las mujeres víctimas de violencia doméstica, las mujeres mayores, las discapacitadas, las refugiadas, las viudas, las pertenecientes a minorías étnicas, las trabajadoras migrantes, las trabajadoras sexuales, las lesbianas y transexuales, las separadas y divorciadas, y las víctimas de desalojos forzosos.

Numerosos estudios llevados a cabo fuera de la esfera de Naciones Unidas coinciden con este diagnóstico como, por ejemplo, los llevados a cabo en América Latina (p. ej. CLADEM/ACCD, 2008; COHRE, 2010); Australia (p. ej. Alice STOAKES; Anitra NELSON, 2005; Selina, TUALLY; Andrew

' Se emplea el término monomarental en lugar de monoparental dado que es una cuestión esencialmente femenina. En España, según datos del Censo de Población y Viviendas de 2011 , en un $79 \%$ de los casos se trata de madres con hijos o hijas a su cargo. 
BEER; Debbie FAULKNER, 2007), u otros de alcance global (p. ej. Ingrid WESTENDOP, 2007; Patricia KENNETT; Chan KAM, 2010).

\section{La exclusión residencial de la mujer en España (2008-2017)}

En España, la situación es muy similar a la del contexto internacional. El reconocimiento jurídico-institucional del derecho de la mujer a la vivienda, bien a través de los tratados y convenciones internacionales antes vistos que son de aplicación en el territorio español, bien por su inclusión en la legislación nacional, es incuestionable, pero la realidad es que un amplio segmento de la población, en el que se encuentran muchas mujeres, sufre exclusión residencial.

Y, desafortunadamente, esta problemática se ha acentuado desde el estallido de la crisis. A lo largo del periodo 2008-2013, el esfuerzo económico a la vivienda, es decir, la proporción de ingresos que se destina a satisfacer los costes de la vivienda ${ }^{2}$, subió con fuerza en España y, pese a disminuir levemente entre los años 2013-2017, el de las mujeres siempre fue mayor durante todo este intervalo de tiempo (Gráfico 1).

Gráfico 1 - Evolución de la mediana de la distribución del porcentaje del total de costes de la vivienda respecto los ingresos del hogar por sexo, España 2008-2017.

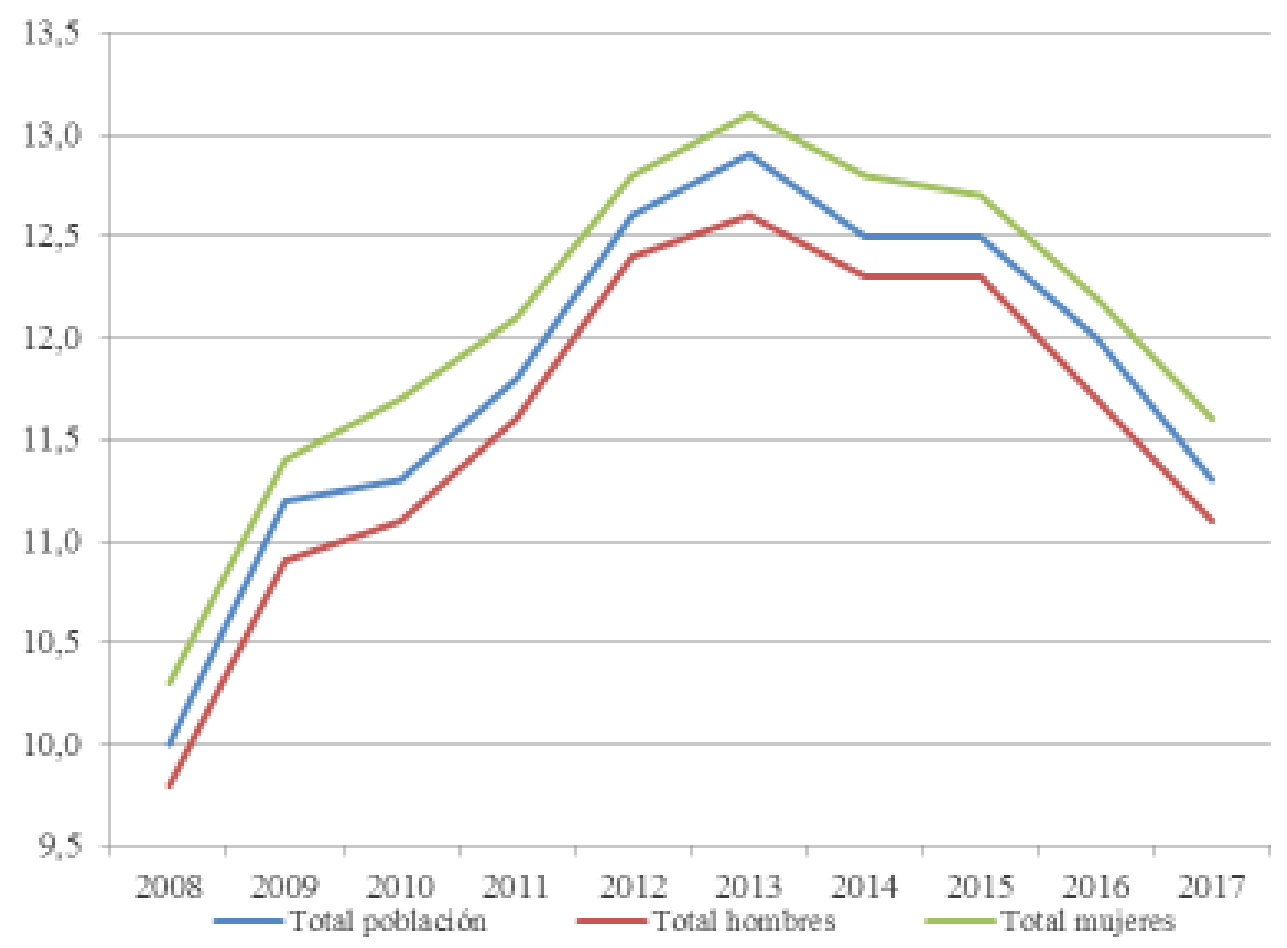

Fuente: European Survey on Income and Living Conditions (EU-SILC), EUROSTAT.

\#PraTodoMundoVer Gráfico de línea, con los años en el eje horizontal, desde 2008 hasta 2017, y el porcentaje del coste de la vivienda respecto los ingresos en el eje vertical. Consta de tres líneas, una de color azul que representa los resultados del total de la población, una de color rojo que muestra los de los hombres, y una verde de las mujeres. La evolución de estas tres líneas es prácticamente paralela, dibujando una silueta de montaña, con el pico en el 2013, y siempre la línea verde por encima de las otras dos.

La monomarentalidad ${ }^{3}$, el alquiler, y los hogares unipersonales son variables que se asocian también con una peor relación entre costes residenciales e ingresos del hogar. El esfuerzo económico medio en el mercado de alquiler que deben realizar los hogares monomarentales y los unipersonales se ha situado a lo largo del periodo 2008-2017, por lo general, por encima del $40 \%$ de los ingresos (Gráfico 2), es decir, supera el umbral máximo por encima del cual se considera muy probable que una familia situada en los dos quintiles inferiores de renta no disponga de recursos suficientes para otras necesidades básicas como la comida, el transporte o la ropa (p. ej. CIHI, 2004; CHP, 2005, 2006; Howard CAMPBELL; Joan MCFADDEN, 2006). Al respecto, es importante tener presente que el

\footnotetext{
${ }^{2}$ Los costes residenciales están formados por el alquiler o la cuota hipotecaria sin la parte correspondiente a amortización del crédito hipotecario (la metodología de EUROSTAT considera este gasto ligado a un bien de consumo de naturaleza duradera), y por la totalidad del coste de los servicios de la vivienda, a saber, el servicio de agua, el impuesto de bienes inmuebles, la electricidad, el alcantarillado, el seguro del hogar, la recogida de basuras y otros impuestos municipales.

${ }^{3}$ Véase nota 1 .
} 
esfuerzo económico medio de la población española que vive en alquiler es uno de los más elevados de toda Europa (Jordi BOSCH, 2015, p. 168).

Gráfico 2- Evolución de esfuerzo económico medio a la vivienda por tipo de hogar, régimen de tenencia de la vivienda y sexo, España 2008-2017.

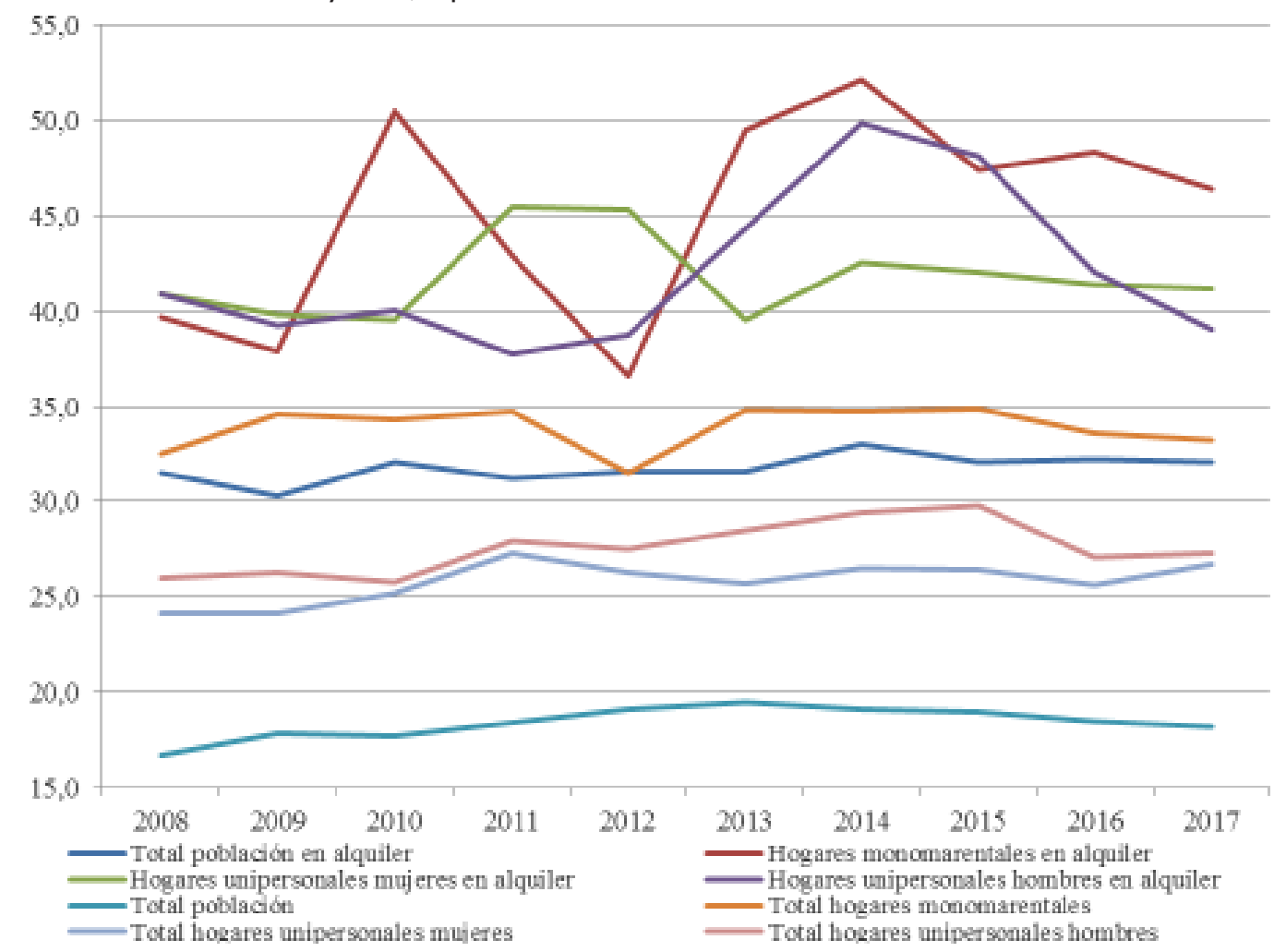

Fuente: European Survey on Income and Living Conditions (EU-SILC), EUROSTAT.

\#PraTodoMundoVer Gráfico de línea, con los años en el eje horizontal, desde 2008 hasta 2017, y el esfuerzo económico medio a la vivienda en porcentaje de los ingresos por tipo de hogar en el eje vertical. Consta de ocho líneas, correspondientes a los siguientes tipos de hogar y tenencia de la vivienda: total población, población en alquiler, hogares unipersonales de hombres, hogares unipersonales de mujeres, hogares unipersonales de hombres en alquiler, hogares unipersonales de mujeres en alquiler, hogares monomarentales, y hogares monomarentales en alquiler.

No obstante, al contrario de lo que cabría esperar, el esfuerzo económico medio de los hogares unipersonales masculinos es ligeramente superior al de los femeninos, entre uno y cuatro puntos mayor (Gráfico 2). El principal factor que explica tal circunstancia son los mayores costes residenciales de los hombres que viven solos en comparación con los de las mujeres. Según datos de EUROSTAT (EU-SILC), entre los años 2008 y 2017, el coste de la vivienda de los hogares unipersonales masculinos fue, de media, un $22 \%$ superior al de los femeninos. De ello se deduce, por un lado, que los ingresos de los hombres que viven solos son mayores al de las mujeres y, por tanto, pueden hacer frente a costes y esfuerzos económicos a la vivienda superiores; $y$, por otro, que las mujeres que viven solas, en mayor proporción, lo hacen en el segmento del parque más económico y, previsiblemente, con un nivel de equipamiento y superficie menor. En este sentido, los resultados del módulo específico de vivienda de la Encuesta de Condiciones de Vida de 2012 sobre satisfacción residencial son reveladores. En 2012, un 12,9\% de las mujeres manifestaba sentirse insatisfecha o muy insatisfecha con su vivienda, frente al 11,2\% de los hombres. Del mismo modo, también el porcentaje de mujeres que ese mismo año afirmaba sufrir falta de espacio en su vivienda era mayor entre las mujeres que entre los hombres. Tales resultados son coherentes con estudios anteriores. En 2006, en su estudio basado en el Censo de 2001, Bosch denunciaba que, en España:

[...] las condiciones residenciales de los hogares encabezados por una mujer son, comparativamente y por lo general, peores: viviendas más antiguas, peor equipadas y conservadas y con mayor tendencia al alquiler como régimen de tenencia asociado a la precariedad socioeconómica. (BOSCH, 2006, p. 36)

Los elevados costes residenciales que deben cubrir los inquilinos también ayudan a entender el porqué de su mayor esfuerzo económico. Entre 2008 y 2017 se situó en torno a los 600 euros, 
mientras que el de los propietarios, habida cuenta que muchos de ellos no tienen pagos hipotecarios pendientes ${ }^{4}$, osciló únicamente alrededor de los 300 euros.

La evolución de la tasa de sobreesfuerzo económico a la vivienda, a saber, del porcentaje de población cuyos costes residenciales son superiores al $40 \%$ de los ingresos del hogar, confirma la crisis que sufre una creciente parte de la sociedad española. Entre 2008 y 2014 , dicho indicador tuvo una clara tendencia alcista, y solo fue a partir de 2014 que empezó a remitir paulatinamente (Gráfico 3). Por sexos, el porcentaje de mujeres en situación de sobreesfuerzo económico fue siempre superior al de los hombres a lo largo de este periodo, excepto durante el bienio 2013-2014.

Gráfico 3- Evolución la población en situación de sobreesfuerzo económico a la vivienda por sexo, España 2008-2017.

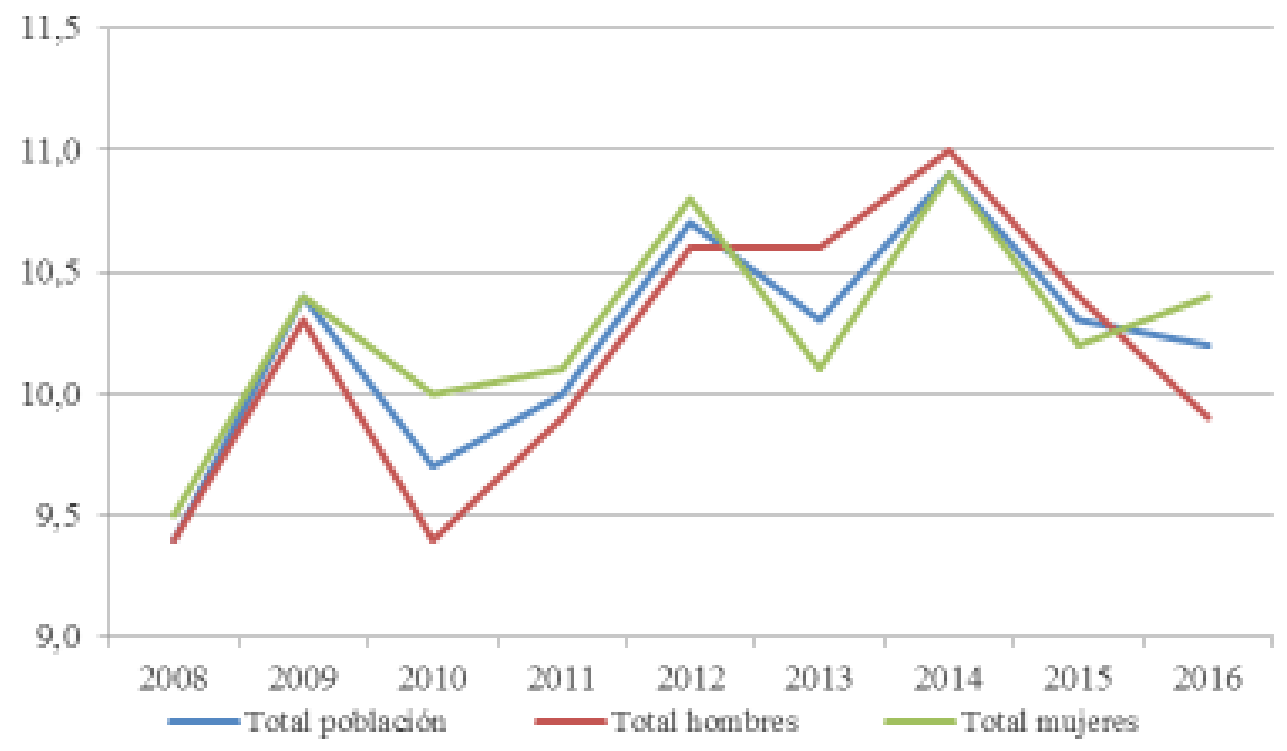

Fuente: European Survey on Income and Living Conditions (EU-SILC), EUROSTAT.

\#PraTodoMundoVer Gráfico de línea, con los años en el eje horizontal, desde 2008 hasta 2017, y el porcentaje de población en situación de sobreesfuerzo económico a la vivienda. Consta de tres líneas, una de color azul que representa los resultados del total de la población, una de color rojo que muestra los hombres, y una verde de las mujeres. Excepto entre los años 2013 y 2015 , durante el resto de los años la línea verde se sitúa por encima de las otras dos.

Existen dos problemáticas de las cuales la información estadística disponible es muy limitada para llevar a cabo un análisis en profundidad: las necesidades residenciales de las mujeres víctimas de violencia de género, y las mujeres afectadas por ejecuciones hipotecarias y procesos de desahucio. Respecto la primera, solo podemos conocer el número de mujeres afectadas gracias a la Estadística de Violencia Doméstica y Violencia de Género (INE). Entre el 2011 y el 2017, el número de víctimas de violencia de género ${ }^{5}$ osciló entre las 27 mil y las 32 mil mujeres por año, y el de víctimas de violencia doméstica, alrededor de las 7 mil anuales, de las cuales más del $60 \%$ eran mujeres. De todo ello se desprende la existencia de una demanda constante de soluciones habitacionales seguras para buena parte de estas mujeres.

Respecto los desahucios y las ejecuciones hipotecarias, pese a la ausencia de estadísticas oficiales desagregadas por sexos, en los últimos años un creciente número de estudios han analizado esta grave problemática. Amnistía Internacional España (2017) considera que, en el parque de alquiler, la expansión de los desahucios ha afectado de forma especial a las mujeres a causa de las múltiples formas de discriminación que sufren, sobre todo las madres solteras, las mujeres que se encargan del cuidado de otras personas, las inmigrantes, las mujeres con discapacidad y las víctimas de violencia de género. En esta misma línea, Maria Inmaculada Sánchez (2015), tras examinar las quejas recibidas por el Defensor del Pueblo Andaluz, detectó un incremento notable de los desahucios, ya sea por impago del crédito hipotecario o del alquiler, entre los hogares encabezados por una mujer con cargas familiares. De igual modo, Amaia

\footnotetext{
${ }^{4}$ Según datos de la Encuesta Continua de Hogares, en 2015 , un 48\% del total de hogares de España vivía en una vivienda de su propiedad sin pagos pendientes.

${ }^{5}$ Esta encuesta considera violencia de género todo acto de violencia física o psicológica (incluidas las agresiones a la libertad sexual, las amenazas, las coacciones o la privación arbitraria de libertad) que se ejerza contra una mujer por parte del hombre que sea o haya sido su cónyuge o esté o haya estado ligado a ella por una relación similar de afectividad aún sin convivencia.
} 
García y Aitziber Ełxezarreta (2018) constataron en su estudio centrado en Araba que las mujeres sufren en mayor medida procesos de ejecución hipotecaria y desahucios. Human Rights Watch también coincide con este diagnóstico. En su opinión, las mujeres cabeza de familia y las que están atadas por sus hipotecas a una expareja maltratadora u hostil constituyen dos grupos particularmente vulnerables en esta crisis (Judith SUNDERLAND, 2014).

Los estudios llevados a cabo desde la Plataforma de Afectados por la Hipotecá también son concluyentes al respecto. Según su principal trabajo cuantitativo sobre el perfil sociológico de los hogares afectados por ejecuciones hipotecarias en España (ODESC/PAH, 2013), un 53\% de éstos está compuesto por padres y/o madres con hijos a su cargo, y un 30\% tenía una o más personas mayores a su cargo, de lo que cabe deducir una mayor proporción de mujeres entre las personas afectadas dado su rol en el hogar como cuidadora, y por la feminización de la vejez. Trabajos posteriores de ámbito regional y local, como los llevado a cabo en Cataluña y Barcelona respectivamente (ODESC/PAH, 2015, 2016, 2018) han confirmado esta feminización de la exclusión residencial, no solo en cuestión de lanzamientos y ejecuciones hipotecarias, sino también en otros aspectos como la pobreza energética.

Pero, además, desde la perspectiva de la salud pública, los desahucios son un grave problema que con mayor intensidad perjudica a la mujer. Investigaciones recientes han constatado que el impacto adverso que tienen los procesos de desahucio en la salud de las personas afectadas es mayor entre las mujeres que entre los hombres (Julia BOLÍVAR et al., 2016; ODESC/PAH, 2015, 2018).

\section{Causas de la mayor exclusión residencial de la mujer}

Esta peor posición de las mujeres en el mercado de vivienda español se debe, fundamentalmente a: (i) la discriminación que sufren en el mercado laboral; (ii) la extrema debilidad de la política de vivienda llevada a cabo en España; (iii) y la insuficiencia de las medidas implementadas para promover la igualdad entre hombres y mujeres.

\section{La discriminación laboral de la mujer}

La situación económica de los individuos en el mercado residencial está fuertemente determinada por su capacidad para obtener rendimientos del trabajo y, lamentablemente, todos los indicadores confirman la peor integración de las mujeres en el mercado laboral español en comparación con la de los hombres (Tabla 1): (i) la tasa de actividad femenina sigue siendo inferior a la masculina; (ii) la tasa de desempleo y de temporalidad son sistemáticamente mayores entre las mujeres que entre los hombres; y (iii) la diferencia salarial entre sexos sigue siendo claramente favorable a los hombres. Es ampliamente conocido que las mujeres tienen menos oportunidades para promocionar laboralmente, perciben sueldos inferiores que los hombres por realizar tareas idénticas, y en mayor proporción trabajan a tiempo parcial, en muchos casos, a causa de las dificultades que encuentran para conciliar vida laboral y familiar puesto que son, abrumadoramente, las que se encargan en el ámbito familiar del cuidado de las personas mayores, de los niños y de otros familiares dependientes, y también asumen en mayor proporción las tareas domésticas (AMNISTÍA INTERNACIONAL ESPAÑA, 2015, 2017). Además, una consecuencia directa de esta menor y peor calidad de la participación de la mujer en el mercado laboral son las inferiores prestaciones por desempleo y pensiones que percibe (Tabla 1), lo cual favorece la cronificación del riesgo de exclusión de la mujer a lo largo de su vida.

Lógicamente, esta inferior posición de las mujeres en los mercados de trabajo y en materia de pensiones es la razón primordial de la feminización de la exclusión social en España. En 2014, un $60,8 \%$ de la población atendida por Cruz Roja en toda España en los programas de Intervención Social eran mujeres (Graciela MALGESINI, 2015, p. 10). De igual modo, a la luz de los datos estadísticos, el VII Informe Foessa sobre exclusión y desarrollo social en España advirtió que "Ios hogares encabezados por una mujer siguen estando más afectados por la exclusión” (Francisco LORENZO, 2014, p. 164), y "enfrentan un riesgo de vivir procesos de exclusión considerablemente más elevados" (p. 165). Asimismo, respecto los diferentes tipos de hogares encabezados por mujeres, existe una amplia unanimidad sobre la penosa situación en que se encuentran los hogares monomarentales (LORENZO, 2014; Javier Eloy MARTíNEZ; Anastasia TÉLLEZ, 2016), circunstancia particularmente preocupante dado que este tipo de hogar ha crecido exponencialmente en España en los últimos 30 años, representando en la actualidad casi el 10\% del total de hogares. Por ejemplo, los valores más elevados del Indicador Global de Vulnerabilidad desarrollado por Cruz Roja recaen sobre los hogares monomarentales (MALGESINI, 2015, p. 35). Otro estudio llevado a cabo también por Cruz Roja Española sobre una muestra de 1051 mujeres de entre 18 y 65 años

\footnotetext{
${ }^{6}$ La Plataforma de Afectados por la Hipoteca es una asociación creada en 2009 que agrupa principalmente a personas con dificultades para pagar la hipoteca o que se encuentran en proceso de ejecución hipotecaria y cuyo cometido, además de prestar apoyo emocional y material a las personas afectadas, es el de denunciar y defender política y jurídicamente el derecho a la vivienda.
} 
representativa de la población atendida arrojaba conclusiones similares: los hogares monomarentales, con el 37,3\% del total, estaban fuertemente sobre representados, y de éstos, un tercio de las madres no recibía ninguna pensión de alimentos por parte del padre (CRUZ ROJA ESPAÑOLA, 2015).

Tabla 1- Indicadores laborales por sexo. España, 2008-2017.

\begin{tabular}{|c|c|c|c|c|c|c|c|c|c|c|}
\hline & 2008 & 2009 & 2010 & 2011 & 2012 & 2013 & 2014 & 2015 & 2016 & 2017 \\
\hline \multicolumn{11}{|c|}{$\begin{array}{l}\text { Tasa de actividad } \\
\text { laboral (1) }\end{array}$} \\
\hline Hombres & 69,5 & 68,7 & 71,1 & 67,4 & 66,9 & 66,2 & 65,8 & 65,7 & 65,1 & 64,7 \\
\hline Mujeres & 50,5 & 51,6 & 55,3 & 52,9 & 53,4 & 53,3 & 53,7 & 53,7 & 53,6 & 53,2 \\
\hline \multicolumn{11}{|c|}{$\begin{array}{l}\text { Salario bruto medio } \\
\text { anual(miles de euros) (2) }\end{array}$} \\
\hline Hombres & 24,2 & 25,0 & 25,5 & 25,7 & 25,7 & 25,7 & 25,7 & 26.0 & 25,9 & n.d. \\
\hline Mujeres & 18,9 & 19,5 & 19,7 & 19,8 & 19,5 & 19,5 & 19,7 & 20,1 & 20,1 & n.d. \\
\hline \multicolumn{11}{|c|}{$\begin{array}{l}\text { Porcentaje de personas } \\
\text { asalariadas con contrato } \\
\text { a tiempo parcial ( } 3 \text { ) }\end{array}$} \\
\hline Hombres & 4,0 & 4,9 & 5,3 & 5,9 & 6,6 & 7,8 & 7,8 & 7,9 & 7,8 & 7,3 \\
\hline Mujeres & 21,9 & 22,4 & 22,7 & 22,9 & 23,9 & 25,3 & 25,6 & 25,2 & 24,1 & 24,2 \\
\hline \multicolumn{11}{|c|}{$\begin{array}{l}\text { Porcentaje de personas } \\
\text { asalariadas con contrato } \\
\text { temporal ( } 1 \text { ) }\end{array}$} \\
\hline Hombres & 27,6 & 23,5 & 23,6 & 24,0 & 22,0 & 22,2 & 23,5 & 25,1 & 25,7 & 25,9 \\
\hline Mujeres & 31,4 & 27,2 & 26,1 & 26,5 & 24,9 & 24,1 & 24,5 & 25,2 & 26,4 & 27,5 \\
\hline \multicolumn{11}{|l|}{ Tasa de paro (1) } \\
\hline Hombres & 10,1 & 17,7 & 19,8 & 21,2 & 24,7 & 25,9 & 23,6 & 20,8 & 18,1 & 15,7 \\
\hline Mujeres & 13,0 & 18,4 & 20,5 & 22,2 & 25,4 & 27,2 & 25,4 & 23,6 & 21,4 & 19,0 \\
\hline \multicolumn{11}{|c|}{$\begin{array}{l}\text { Prestación anual media } \\
\text { por desempleo (euros) (4) }\end{array}$} \\
\hline Hombres & 3.488 & 4.396 & 4.269 & 3.984 & 4.166 & 4.148 & 3.690 & 3.304 & 3.179 & 3.087 \\
\hline Mujeres & 3.044 & 3.513 & 3.559 & 3.357 & 3.475 & 3.450 & 3.188 & 2.962 & 2.882 & 2.850 \\
\hline \multicolumn{11}{|c|}{$\begin{array}{l}\text { Pensión media anual } \\
\text { (miles de euros) (4) }\end{array}$} \\
\hline Hombres & 13,1 & 13,3 & 13,7 & 14,3 & 14,6 & 15,1 & 15,3 & 15,8 & 16,2 & 16,6 \\
\hline Mujeres & 9,0 & 9,3 & 9,7 & 10,1 & 10,4 & 10,7 & 10,9 & 11,0 & 11,3 & 11,7 \\
\hline
\end{tabular}

Fuente: elaboración propia a partir de la Encuesta de Población Activa (1), Encuesta de estructura salarial (2), The European Union Labour Force Survey (EU-LFS) (3); Agencia Tributaria (4).

Fuente: elaboración propia a partir de la Encuesta de Población Activa (1), Encuesta de estructura salarial (2), The European Union Labour Force Survey (EU-LFS) (3); Agencia Tributaria (4).

\#PraTodoMundoVer Tabla cuyas columnas representan cada uno de los años del periodo 2008-2017, y en las filas aparecen diferenciados por sexos los valores correspondientes a la tasa de actividad laboral, el salario bruto medio anual, el porcentaje de personas asalariadas con contrato a tiempo parcial, el de personas asalariadas con contrato temporal, la tasa de paro, la prestación anual media por desempleo y la pensión media anual.

\section{La debillidad de la política de vivlenda}

En el contexto europeo, los principales instrumentos dirigidos a resolver los problemas de accesibilidad económica de la demanda son dos: la vivienda social ${ }^{7}$, y las ayudas directas a las personas en forma de prestaciones económicas o similares para el pago del alquiler o la compra de la vivienda.

España, con tan solo un $2 \%$ de vivienda social se sitúa, juntamente con Grecia, Chipre, Letonia y Estonia, entre los países comunitarios con un menor desarrollo de este instrumento de política de vivienda (Elsa LAINO; Alice PITTINI, 201 1). Igualmente, el sistema de ayudas al pago de la vivienda español es uno de los más débiles del continente: solo se destina a este fin un $0,2 \%$ del PIB, apenas un $1,4 \%$ de la población se beneficia de dichas ayudas y, al contrario de muchos países comunitarios, se centra en facilitar el acceso a la propiedad en lugar de atender a la población que vive de alquiler (BOSCH, 2017).

\footnotetext{
${ }^{7}$ En el ámbito político y académico europeo, la vivienda social es aquella de alquiler asequible, de titularidad pública o de entidades sin ánimo de lucro, y cuya provisión no se realiza dentro de los mecanismos del mercado, sino que es el resultado de procesos impulsados directa o indirectamente por el sector público con el fin de atender las necesidades residenciales de la población con problemas de accesibilidad económica.
} 
Esta debilidad estructural de la política de vivienda española se ha agravado aún más tras los ajustes presupuestarios que ha realizado el gobierno central con la crisis. Desde el año 2009 el gasto público del gobierno central en materia de acceso a la vivienda y fomento de la edificación ha disminuido alrededor de un $65 \%$, tanto en valores absolutos como en porcentaje sobre el total del gasto público (Tabla 2). Uno de los programas que se ha visto afectado por estos recortes ha sido la Renta Básica de Emancipación. En 2012, dejaron de concederse nuevas ayudas para el pago del alquiler de las personas jóvenes, y las reconocidas anteriormente redujeron su importe en un 30\%. Asimismo, el plan estatal ${ }^{8}$ de vivienda 2013-2016 puso fin al sistema de subvenciones, incentivos y ayudas para la construcción y compra de las denominadas viviendas de protección oficial, una forma de vivienda asequible mayoritariamente de compraventa promovida tanto por promotores privados como públicos que desde mediados del siglo XX hasta el estallido de la crisis constituyó el principal instrumento de la política de vivienda en España (Joris HOEKSTRA; Iñaki HERAS; ETXEZARRETA, 2010; Baralides ALBERDI, 2014). En buena lógica, sin apoyo del Estado, la construcción de vivienda protegida se ha desplomado paulatinamente hasta situarse en unas 5 mil unidades anuales finalizadas en 2017 (Tabla 2), el valor más bajo registrado, como mínimo, desde el año 1960.

Tabla 2 - Indicadores de política de vivienda, España, 2008-2017.

Gasto público imputado al "acceso a la vivienda y fomento de la edificación" en los presupuestos generales del Estado (1)

\begin{tabular}{|c|c|c|c|c|c|c|c|c|c|c|}
\hline & 2008 & 2009 & 2010 & 2011 & 2012 & 2013 & 2014 & 2015 & 2016 & 2017 \\
\hline Total (millones $€$ ) & 1.372 & 1.607 & 1.491 & 1.209 & 820 & 755 & 820 & 587 & 587 & 466 \\
\hline $\begin{array}{l}\text { Porcentaje respecto } \\
\text { total gasto público }\end{array}$ & 0,39 & 0,44 & 0,43 & 0,38 & 0,23 & 0,20 & 0,23 & 0,17 & 0,17 & 0,14 \\
\hline Vivienda protegida (2) & & & & & & & & & & \\
\hline $\begin{array}{l}\text { Número viviendas } \\
\text { protegidas completadas }\end{array}$ & 68.587 & 67.904 & 58.31 & 58.308 & 53.33 & 17.0 & 15. & 7.931 & 7.118 & 4.938 \\
\hline
\end{tabular}

Fuentes: Ministerio de Economía y Hacienda (1) y Ministerio de Fomento (2).

Fuentes: Ministerio de Economía y Hacienda (1) y Ministerio de Fomento (2).

\#PraTodoMundoVer Tabla cuyas columnas representan cada uno de los años del periodo 2008-2017, y las filas los valores de tres indicadores de política de vivienda: el total del gasto público imputado al "acceso a la vivienda y fomento de la edificación" en los presupuestos generales del Estado, el porcentaje de dicho gasto respecto el total del gasto público, y el número de viviendas protegidas completadas.

\section{La insuficiencia de las políticas de igualdad}

Las políticas de austeridad adoptadas tras el inicio de la crisis provocaron un profundo recorte de las políticas sociales, especialmente intenso en materia de igualdad y protección de las mujeres ante la violencia, hasta el punto de suponer, en opinión de numerosas expertas en la materia, su práctico desmantelamiento (p. ej. Yolanda BETETA, 2013; Maria Luz RODRÍGUEZ, 2013; Emanuela LOMBARDO; Margarita LEÓN, 2014).

Este retroceso es también visible en materia de vivienda. El plan de igualdad de oportunidades 2014-2016 (MINISTERIO DE SANIDAD, SERVICIOS SOCIALES E IGUALDAD, 2014) no hace ninguna referencia a las necesidades residenciales de la mujer, pese a que el anterior reconocía explícitamente que había que "Introducir o ampliar, en su caso, la perspectiva de género en los criterios de adjudicación de viviendas protegidas y de acceso a ayudas económicas al pago de alquiler" (MINISTERIO DE TRABAJO Y ASUNTOS SOCIALES, 2007). No olvidemos que la vigente ley marco en materia de igualdad, la Ley Orgánica 3/2007, de 22 de marzo, para la igualdad efectiva de mujeres y hombres, estableció hace diez años que las políticas y planes de las Administraciones públicas en materia de acceso a la vivienda debían incluir medidas destinadas a hacer efectivo el principio de igualdad entre mujeres y hombres, para lo cual exhortaba al gobierno a que, en el ámbito de sus competencias, fomentara el acceso a la vivienda de las mujeres en situación de necesidad o en riesgo de exclusión, y de las víctimas de la violencia de género, en especial cuando, en ambos casos, tuvieran hijos menores a su cargo.

Asimismo, la legislación estatal sobre política de vivienda apenas contempla disposiciones en materia de perspectiva género. La única medida destacable del plan de vivienda 2013-2016 es la inclusión de las víctimas de violencia doméstica como uno de los sectores preferentes en la concesión de las escasas ayudas existentes.

\footnotetext{
${ }^{8}$ Real Decreto 233/2013, de 5 de abril, por el que se regula el Plan Estatal de fomento del alquiler de viviendas, la rehabilitación edificatoria, y la regeneración y renovación urbanas, 2013-2016. Este plan estatal de vivienda, conjuntamente con la Ley $4 / 2013$, de 4 de junio, de medidas de flexibilización y fomento del mercado del alquiler de viviendas suprimieron las ayudas a los adquirientes de vivienda protegida, entre las que se incluía la subsidiación de los préstamos para su compra.
} 
Del mismo modo, el alcance de la perspectiva de género en las diferentes disposiciones ${ }^{9}$ que ha aprobado el gobierno central para proteger a los deudores hipotecarios es ínfimo. Para poder gozar de beneficios como la dación en pago, tipos de interés inferiores para moderar el crecimiento de la deuda hipotecaria, poder renegociar o suprimir parte de la deuda, o disfrutar de moratorias para la ejecución hipotecaria, desde una perspectiva de género, solamente se considera como circunstancia familiar de especial vulnerabilidad, la unidad familiar monomarental con dos hijos a cargo, la unidad de la que forme parte un menor de tres años, y las víctimas de violencia de género. Además, la normativa fija unos requisitos fuertemente restrictivos en materia de ingresos del hogar, coste de la vivienda y composición familiar, lo cual ha reducido el número de potenciales destinatarios. Entre marzo de 2012 y junio de 2014, de las cerca de 24 mil familias que trataron de acogerse a alguno de estos beneficios, sólo 2.124 consiguieron la dación en pago.

A nivel autonómico, es habitual que los planes de vivienda de las comunidades incluyan medidas de género como el acceso prioritario de los hogares monomarentales y de las víctimas de violencia doméstica a una vivienda de protección oficial o a las ayudas disponibles para el pago del alquiler (Montse PAREJA-EASTWAY; Teresa SANCHEZ-MARTINEZ, 2010). Sin embargo, la efectividad de éstas está condicionada, sobre todo, por la disponibilidad de vivienda social y de ayudas económicas suficientemente dotadas en los correspondientes presupuestos y, como veíamos anteriormente, el desarrollo de ambos instrumentos es mínimo. En otros casos, los obstáculos son de tipo jurídico-burocrático. A pesar de que la Ley Orgánica 1/2004, de 28 de diciembre, de medidas de protección integral contra la violencia de género concede un derecho de acceso prioritario a viviendas protegidas a las mujeres víctimas de violencia de género (María Nieves PACHECO, 2015), es usual que para acceder a ella se exija: (i) que las víctimas de violencia de género tengan una orden de protección, lo cual dilata en el tiempo el trámite, con los consiguientes riesgos que eso entraña para la víctima; (ii) o no tener una vivienda en propiedad, problema muy común que impide a las víctimas alejarse de su agresor.

\section{Conclusiones y discusión}

El estallido de la burbuja residencial y la posterior recesión económica provocaron una grave crisis social en España que, aún lejos de resolverse, ha afectado con mayor intensidad a las mujeres. La vivienda es uno de los campos donde es más visible esta feminización de la exclusión social. Entre los años 2008 y 2013 , la relación entre ingresos del hogar y costes residenciales empeoró de forma notable, y siempre fue más desfavorable para las mujeres. Este deterioro de la capacidad de los hogares para satisfacer los costes de su vivienda generó una escalada del número de desahucios que, según todos los indicios, ha tenido una mayor incidencia entre los hogares encabezados por una mujer, sobre todo entre los hogares monomarentales, entre las mujeres que viven solas en alquiler, y entre las que deben afrontar elevados pagos hipotecarios.

Asimismo, pese a tratarse de problemáticas de las que no se dispone de suficiente información estadística para poder ser examinadas en profundidad, de acuerdo con la literatura existente es muy probable que las mujeres víctimas de violencia doméstica, las trabajadoras sexuales y las víctimas de explotación sexual, las mujeres expresas, las pertenecientes a minorías étnicas, las discapacitadas, las trabajadoras migrantes, y las separadas y divorciadas, también hayan sufrido en mayor proporción y gravedad esta crisis residencial. En este sentido, es importante subrayar la necesidad de mejorar la estadística disponible sobre las diferentes formas de exclusión residencial, tanto a nivel cuantitativo como desde un enfoque de género, para poder formular diagnósticos más precisos de la problemática y, en consecuencia, poder planificar adecuadamente las medidas necesarias y efectuar su oportuno seguimiento.

Esta peor situación de la mujer en el mercado de vivienda español se debe, fundamentalmente, a la discriminación que sufre en el mercado laboral. Su capacidad para obtener rentas del trabajo es muy inferior a la de los hombres y, en consecuencia, también las pensiones que percibe por desempleo o jubilación.

Lamentablemente no existen medidas que corrijan esta inferior posición de la mujer. Los recortes de gasto público que se han llevado a cabo tras la crisis han provocado el práctico desmantelamiento de las políticas de igualdad y han debilitado aún más la escasa intervención pública en vivienda. La caída de la construcción de vivienda protegida hasta unos niveles mínimos históricos es una buena muestra de ello. Además, el enfoque de género de la política de vivienda española es ínfimo, centrado casi exclusivamente en los hogares monomarentales y en las víctimas de violencia de género.

En este contexto, resulta pertinente preguntarse por el papel efectivo de los numerosos tratados y resoluciones internacionales que exhortan a los gobiernos a garantizar el derecho de la

\footnotetext{
${ }^{9}$ Real Decreto-ley 27/2012, de 15 de noviembre, de medidas urgentes para reforzar la protección a los deudores hipotecario; Ley $1 / 2013$, de 14 de mayo, de medidas para reforzar la protección de los deudores hipotecarios, reestructuración de deuda y alquiler social; y Real Decreto-ley 1/2015, de 27 de febrero, de mecanismo de segunda oportunidad, reducción de carga financiera y otras medidas de orden social.
} 
mujer a una vivienda digna y adecuada. ¿Cómo puede explicarse sus elevadas cotas de vulneración a pesar de la extensa relación de tratados y convenciones internacionales que lo protegen y que son de aplicación en el territorio español? Probablemente, el principal problema radica en la distancia existente entre el plano teórico-declarativo (politics) y el práctico-político (policy). Una de las críticas más recurrentes a las conferencias Hábitat de Naciones Unidas es la ausencia de directrices claras sobre su implementación, de compromisos económicos para su ejecución, y de mecanismos de seguimiento y evaluación del cumplimiento de los objetivos (Michael COHEN, 2016), lo que acaba confiriendo a las resoluciones de estas conferencias un carácter meramente declarativo. Igualmente, las referencias al género que incluye la Nueva Agenda Urbana aprobada en la conferencia Hábitat III en 2016, aunque positivas, son superficiales y no llegan a representar compromisos transformadores por sí mismas (Inés SÁNCHEZ DE MADARIAGA, 2017 , p. 111). En cierto modo, es lo mismo que sucede con el reconocimiento constitucional del derecho a la vivienda y a la igualdad entre hombres y mujeres. La casi totalidad de las constituciones nacionales, incluida la española, recogen ambos derechos, pero su materialización efectiva queda supeditada al alcance de las políticas públicas implementadas en estos ámbitos. Por todo ello, sin menoscabar el importante papel que estas organizaciones internacionales han jugado en el análisis y denuncia de las diferentes formas de exclusión residencial que sufre la mujer, resulta obvio que, actualmente, el principal reto no consiste en insistir en declaraciones sobre la necesidad de introducir la perspectiva de género en las políticas urbanas, incluidas las de vivienda, sino en conseguir que estas políticas se implementen.

\section{Referencias}

ALBERDI, Baralides. "Social housing in Spain". In: SCANLON, Kathleen; WHITEHEAD, Christine; FERNANDEZ, Melissa (Eds). Social Housing in Europe. Hoboken, New Jersey: John Wiley \& Sons, 2014. p. 223-237.

AMNISTÍA INTERNACIONAL ESPAÑA. Derechos desalojados. El derecho a la vivienda y los desalojos hipotecarios en España. Madrid: Amnistía Internacional España, 2015.

AMNISTÍA INTERNACIONAL ESPAÑA. La crisis de vivienda no ha terminado. El derecho a la vivienda y el impacto de los desahucios de viviendas de alquiler sobre las mujeres en España. Madrid: Amnistía Internacional España, 2017.

BETETA, Yolanda. "La feminización de la crisis financiera global. La regresión del estado de bienestar en España y su impacto en las políticas de igualdad y de erradicación de la violencia contra las mujeres. Nuevos retos". Asparkía: investigació feminista, Castellón de la Plana, n. 24, p. 36-52, jan./ dez.2013.

BOLÍVAR, Julia et al. "La salud de las personas adultas afectadas por un proceso de desahucio". Gaceta Sanitaria, v. 30, n. 1, p. 4-10, jan./fev. 2016.

BOSCH, Jordi. "La dimensión económica de la exclusión residencial: Cataluña en el contexto europeo". ACE: Architecture, City and Environment, Barcelona, v. 12, n. 34, p. 69-96, jun. 2017.

BOSCH, Jordi. Crisis y emancipación juvenil en Europa. Un análisis desde los modelos de Estado de Bienestar. Vitoria-Gasteiz: Servicio Central de Publicaciones del Gobierno Vasco, 2015.

BOSCH, Jordi. El problema de la vivienda en España desde una perspectiva de género: análisis y propuestas para su desarrollo. Madrid: Fundación Alternativas, Estudios de Progreso, 2006.

CAMPBELL, Howard; MCFADDEN, Joan. "Healthy living: Housing affordability and its impact on family Health". Journal of Family and Consumer Sciences, Alexandria, v. 98, n. 4, p. 49-51, nov. 2006.

CGLU. La Carta Europea de Salvaguarda de los Derechos Humanos en la ciudad. Barcelona: Ciudades y Gobiernos Locales Unidos, Comisión de Inclusión Social, Democracia Participativa y Derechos Humanos, 2012.

CHP.A Heavy Load: The Combined Housing and Transportation Burdens of Working Families. Washington, DC: Center for Housing Policy, 2006.

CHP. Something's Gotta Give: Working Families and the Cost of Housing. Washington, DC: Center for Housing Policy, 2005.

CIHI. Housing and Population Health. The State of Current Research Knowledge.Ottawa: Canadian Institute for Health Information, 2004. 
CLADEM/ACCD. Sistematización de Estudios Nacionales sobre Derechos Habitacionales y DESC de las mujeres en Argentina, Bolivia, Brasil, Chile, Colombia, Ecuador, El Salvador, Guatemala, Honduras, México, Panamá, Paraguay, Perú, República Dominicana y Uruguay. Lima: Comité de América Latina y El Caribe para la Defensa de los Derechos de la Mujer (CLADEM) y Agència Catalana de Cooperació al Desenvolupament (ACCD), 2008.

COHEN, Michael. "From Habitat II to Pachamama: a growing agenda and diminishing expectations for Habitat III". Environment and Urbanization, London, v. 28, n. 1, p. 35-48, dez. 2016.

COHRE. Un lugar en el mundo. El derecho a una vivienda adecuada como elemento esencial de una vida libre de violencia doméstica. Los casos de Argentina, Brasil y Colombia. Ginebra: Center on Housing Rights and Evictions (COHRE), 2010.

CRUZ ROJA ESPAÑOLA. Boletín sobre vulnerabilidad social. Madrid: Departamento de Estudios e Innovación Social, Cruz Roja Española, 2015.

EMANUELLI, María Silvia (Ed). Vivienda con rostro de mujer: mujeres y derecho a una vivienda adecuada. México: Social Watch, Coalición Internacional para el Hábitat, Oficina Regional para América Latina (HIC-AL) y Red Mujer y Hábitat América Latina. 2004.

FARHA, Leilani. Informe de la Relatora Especial sobre una vivienda adecuada como elemento integrante del derecho a un nivel de vida adecuado y sobre el derecho de no discriminación a este respecto. Nueva York: Asamblea General de Naciones Unidas, 2015.

GARCÍA, Amaia; ETXEZARRETA, Aitziber. "Un estudio de caso de los desahucios a nivel local: el caso de stop desahucios Araba". Revista de Economía Crítica, Barcelona, n. 25, p. 26-46, jan./jun. 2018.

HOEKSTRA, Joris; HERAS, Iñaki; ETXEZARRETA, Aitziber. "Recent changes in Spanish housing policies: subsidized owner-occupancy dwellings as a new tenure sector?" Journal of Housing and the Built Environment, v. 25, n. 1, p. 125-138, abr. 2010.

KENNETT, Patricia; KAM, Chan (Eds). Women and Housing. Oxon: Taylor \& Francis, 2010.

KOTHARI, Miloon. La mujer y la vivienda adecuada. Informe del Relator Especial sobre una vivienda adecuada como elemento integrante del derecho a un nivel de vida adecuado y sobre el derecho de no discriminación a este respecto. Nueva York: Consejo Económico y Social, Naciones Unidas, 2006.

KOTHARI, Miloon. Women and adequate housing. Study by the Special Rapporteur on adequate housing as a component of the right to an adequate standard of living, and the right to-nondiscrimination. Nueva York: Comisión de Derechos Humanos, Naciones Unidas, 2003.

KOTHARI, Miloon. Women and adequate housing. Study by the Special Rapporteur on adequate housing as a component of the right to an adequate standard of living. Nueva York: Comisión de Derechos Humanos, Naciones Unidas, 2004.

LAINO, Elsa; PITTINI, Alice. Housing Europe Review 2012.The nuts and bolts of European social housing systems. Bruselas: CECODHAS Housing Europe, 2011.

LOMBARDO, Emanuela; LEÓN, Margarita. "Políticas de igualdad de género y sociales en España: origen, desarrollo y desmantelamiento en un contexto de crisis económica". Investigaciones feministas, Madrid, v. 5, p. 13-35, fev. 2014.

LORENZO, Francisco. (Ed). VII Informe sobre exclusión y desarrollo social en España. Madrid: Fundación FOESSA, 2014.

MALGESINI, Graciela. Informe sobre vulnerabilidad social 2014. Madrid: Cruz Roja Española, 2015.

MARTíNEZ, Javier Eloy; TÉLLEZ, Anastasia. "El efecto de la crisis y el desempleo desde una perspectiva de género". Cuestiones de Género: de la igualdad y la diferencia, n. 11 , p. 351-372, jan./dez.2016.

MÉNDEZ, Ricardo. "De la hipoteca al desahucio: ejecuciones hipotecarias y vulnerabilidad territorial en España". Revista de Geografía Norte Grande, Santiago de Chile, n. 67, p. 9-31, set. 2017.

MÉNDEZ, Ricardo; ABAD, Luis; PLAZA, Julio. Geografía de las ejecuciones hipotecarias en España. Madrid: Fundación 1 de Mayo, Colección Estudios, 2014. 
MÉNDEZ, Ricardo; PLAZA, Julio. "Crisis inmobiliaria y desahucios hipotecarios en España: una perspectiva geográfica”. Boletín de la Asociación de Geógrafos Españoles, n. 71, p. 99-127, mai./ ago. 2016.

MINISTERIO DE SANIDAD, SERVICIOS SOCIALES E IGUALDAD. Plan Estratégico de Igualdad de Oportunidades 2014-2016. Madrid: Instituto de la Mujer y para la Igualdad de Oportunidades, 2014.

MINISTERIO DE TRABAJO Y ASUNTOS SOCIALES. Plan estratégico de igualdad oportunidades (20082011). Madrid: Instituto de la Mujer, 2007.

NACIONES UNIDAS. Nueva Agenda Urbana. Quito: Secretariado de Hábitat III, 2017.

ODESC. Mediterranean Regional Consultation on Women's Right to Adequate Housing. Barcelona: Observatorio Derechos Humanos DESC, 2006.

ODESC/PAH. "Impactes de la crisihipotecària en la salut". In: Vanesa VALIÑO (Ed). Emergència habitacional a Catalunya. Impacte de la crisihipotecària en el dret a la salut i elsdretsdelsinfants. Barcelona: Observatorio Derechos Humanos DESC y Plataforma de Afectados por la Hipoteca, 2015. p. 96-103.

ODESC/PAH. Emergencia habitacional en el estado español: la crisis de las ejecuciones hipotecarias y los desalojos desde una perspectiva de derechos humanos. Barcelona: Observatorio Derechos Humanos DESC y Plataforma de Afectados por la Hipoteca, 2013.

ODESC/PAH. Exclusión residencial en el mundo local. Informe de la crisis hipotecaria en Barcelona (2013-2016). Barcelona: Observatorio Derechos Humanos DESC y Plataforma de Afectados por la Hipoteca, 2016.

ODESC/PAH. Radiografies de la situació del dret a l'habitatge, la pobresa energètica i el seu impacte en la salut a Barcelona. Informe II. Perspectiva de gènere sobre el dret a l'habitatge i la pobresa energètica a Barcelona. Barcelona: Observatorio Derechos Humanos DESC y Plataforma de Afectados por la Hipoteca, 2018.

PACHECO, María Nieves. "Evolución y situación actual en España del derecho preferente de acceso a vivienda protegida de las mujeres víctimas de violencia de género". Aequalitas: Revista jurídica de igualdad de oportunidades entre mujeres y hombres, v. 36, p. 6-14, jan./jun. 2015.

PAREJA-EASTWAY, Montse; SANCHEZ-MARTINEZ, Teresa. "Social change and Housing Systems: The Case of Women in Spain". In: KENNETT, Patricia; KAM, Chan (Eds). Women and Housing. Oxon: Taylor \& Francis, 2010. p. 71-95.

RODRÍGUEZ, María Luz. "Claves de género de la crisis económica en España". In: CONGRESO DE LA RED ESPAÑOLA DE POLÍTICA SOCIAL (REPS): LAS POLÍTICAS SOCIALES ENTRE CRISIS Y POST-CRISIS, 4, 2013, Alcalá de Henares (Madrid), Fundación Foessa, Actas del $4^{\circ}$ Congreso, Alcalá de Henares: Universidad de Alcalá/Red Española de Política Social, 2013. p. 428-439.

ROLNIK, Raquel. Informe de la Relatora Especial sobre una vivienda adecuada como elemento integrante del derecho a un nivel de vida adecuado y sobre el derecho de no discriminación a este respecto. Nueva York: Asamblea General de Naciones Unidas, 2011.

SALA, Eduard. "Análisis geográfico de la crisis hipotecaria: la distribución desigual a diferentes escalas". Biblio 3w: Revista Bibliográfica de Geografía y Ciencias Sociales [online]. Barcelona, 2018, v.23, n. 1.256, p. 1-24. Disponible en https://www.raco.cat/index.php/Biblio3w/article/view/ 348601/439839. ISSN 1138-9796. Aceso el 13/06/2019.

SÁNCHEZ DE MADARIAGA, Inés. "Una nova generació de polítiques per a la igualtat efectiva de gènere en l'urbanisme. De la Nova Agenda Urbana a les Directriusd'Ordenaciódel Territori del Pais Basc". In: SERRANO, Begoña; MATEO, Carolina; RUBIO, Alberto (Eds). Gènere i política urbana. Arquitectura i urbanismedes de la perspectiva de gènere. Valencia: Institut Valencià de l'Edificació, 2017. p. 111-132.

SÁNCHEZ, Maria Inmaculada. "Sinhogarismo de las familias monomarentales". In: CONGRESO UNIVERSITARIO INTERNACIONAL INVESTIGACIÓN Y GÉNERO, 5, 2014, Sevilla, Universidad de Sevilla, Aportaciones a la investigación sobre mujeres y género Libro de Actas, Sevilla: Universidad de Sevilla, SIEMUS (Seminario Interdisciplinar de Estudios de las Mujeres de la Universidad de Sevilla), 2015. p. 214-225. 
STOAKES, Alice; NELSON, Anitra. Women and housing policy and research. Melbourne: Australian Housing and Urban Research Institute (AHURI), 2005.

SUNDERLAND, Judith. Sueños rotos. El impacto de la crisis española de la vivienda en grupos vulnerables. Nueva York: Human Rights Watch, 2014.

TUALLY, Selina; BEER, Andrew; FAULKNER, Debbie. Too Big to Ignore: Future Issues for Australian Women's Housing 2006-2025. Melbourne: Australian Housing and Urban Research Institute (AHURI), 2007.

UN-HABITAT. African Regional Civil Society Consultation on Women and Adequate Housing. Nairobi: United Nations Human Settlements Programme, 2002.

UN-HABITAT. Proceedings of the Asia Regional Consultation on 'The Interlinkages between Violence against Women and Women's Right to Adequate Housing'. Nueva Delhi: United Nations Human Settlements Programme, 2004.

UN-HABITAT. Proceedings of the Pacific Regional Consultation on Women's Rights to Adequate Housing and Land. Nadi: United Nations Human Settlements Programme, 2006.

UN-HABITAT. Report on the Central-Asia/Eastern Europe Regional Consultation on Women's Right to Adequate Housing. The interlinkages between multiple discrimination and Women's Right to Adequate Housing. Budapest: United Nations Human Settlements Programme, 2005b.

UN-HABITAT. Women and Housing: Towards Inclusive Cities. Nairobi: UN-Habitat, 2014.

UN-HABITAT. Women's Right to Adequate Housing and Land. Middle East/North Africa Proceedings of the Alexandria Consultation. Alejandría: United Nations Human Settlements Programme, y Housing and Land Rights Network, Habitat International Coalition, 2005a.

UN-HUMAN RIGHTS. Women and the right to adequate housing. Nueva York y Ginebra: The United Nations Office of the High Commissioner for Human Rights, 2012.

WESTENDOP, Ingrid. Women and Housing: Gender Makes A Difference. Cambridge: Intersentia Publishers, 2007.

Jordi Bosch Meda (jordi.bosch@hotmail.es) es Dr. Arquitecto por la Escuela Técnica Superior de Arquitectura de Barcelona, Diplomado en Gestión y Administración Pública y Licenciado en Ciencias Políticas por la Universidad de Barcelona. Es experto en políticas de vivienda y urbanismo, sistemas residenciales, y exclusión residencial. En estos temas, es autor de diez libros y más de treinta artículos y capítulos de libro.

COMO CITAR ESTE ARTÍCULO, DE ACUERDO CON LAS NORMAS DE LA REVISTA:

MEDA, Jordi Bosch. "El derecho de la mujer a la vivienda en España (2008-2017)". Revista Estudios Feministas, Florianópolis, v. 28, n. 1, e54148, 2020.

CONTRIBUCIÓN DE AUTORÍA

No aplicable.

FINANCIACIÓN

No aplicable.

CONSENTIMIENTO DE USO DE IMAGEM

No aplicable.

APROBACIÓN DE COMITÉ DE ÉTICA EN INVESTIGACIÓN

No aplicable.

CONFLICTO DE INTERESES

No aplicable. 


\section{LICENCIA DE USO}

Este artículo está licenciado bajo la Licencia Creative Commons CC-BY Internacional. Con esta licencia se puede compartir, adaptar, crear material para cualquier objetivo, siempre que se le atribuya la autoría.

\section{HISTORIAL}

Recibido el 21/11/2017

Presentado nuevamente el 17/06/2019

Aprobado el 30/09/2019 